\title{
HERWIRI2: Exponentiated Electroweak Corrections in a Hadronic Event Generator
}

\author{
Scott A. Yost ${ }^{* \dagger}$ \\ The Citadel \\ Charleston, SC 29409, USA \\ E-mail: scott.yostecitadel.edu \\ Valerie Halyo ${ }^{\ddagger}$ \\ Princeton University \\ Princeton, NJ 08544, USA \\ E-mail: valerieh@princeton.edu
}

\section{Miroslav Hejna}

Princeton University

Princeton, NJ 08544, USA

E-mail: mhejna@princeton.edu

\section{B.F.L. Ward ${ }^{\S}$}

Baylor University Waco, TX 76798, USA

E-mail: bfl_ward@baylor.edu

Reaching the desired precision level for $W$ and $Z$ processes at the LHC will require a mixture of higher-order QCD and electroweak corrections. HERWIRI2 is a step in implementing QED $\otimes$ QCD exponentiation in a hadronic event generator. This program implements leading electroweak corrections and coherent exclusive exponentiation in a HERWIG environment. We discuss the status of the program, recent tests, and future developments.

36th International Conference on High Energy Physics,

July 4-11, 2012

Melbourne, Australia

\footnotetext{
* Speaker.

${ }^{\dagger}$ This work and its presentation were supported in part by D.o.E. grant DE-PS02-09ER09-01 and grants from The Citadel Foundation.

${ }^{\ddagger}$ Work supported in part by D.o.E grant DE-FG02-91ER40671.

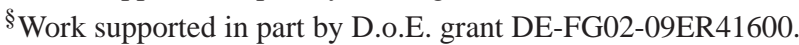


Precise measurements of vector boson production at the LHC will be very important in rigorous testing of the Standard Model. In addition, these production processes have been identified as standard candles for the measurement of the beam luminosity.[1] The desired precision on the theoretical contribution to the error of these measurements is at the $1 \%$ level. Recent studies [2] by some of the authors have found that the state-of-the-art precision tag on single $Z$ production at a CMS energy of $10 \mathrm{TeV}$ is $\sim 4.6 \%$, of which approximately $2 \%$ is due to electoweak corrections. These studies were based on FEWZ[3], which provides NNLO QCD corrections, and HORACE, [4] which provides $\mathscr{O}(\alpha)$ radiative corrections with a final-state photon shower, and PHOTOS, [5] which adds final state photonic radiation.

Attaining $1 \%$ precision will require $\mathscr{O}\left(\alpha_{s}^{2}\right)$ (NNLO) QCD corrections, together with $\mathscr{O}\left(\alpha_{s} \alpha\right)$ electroweak corrections to next-to-leading log, and $\mathscr{O}\left(\alpha^{2}\right)$ to leading log. A general framework based on generalized Yennie-Frautschi-Suura (YFS) exponentiation [6] has been proposed to implement these corrections incrementally in a hadronic event generator which should inherit some of the advantages of YFS-exponentiated Monte Carlo programs developed for LEP physics, such as BHLUMI [7], KKMC [8], and related programs[9]. This framework has been named HERWIRI[10, 11], for "High Energy Radiation With Infra-Red Improvements," and generalizes the YFS approach to encompass both QED and QCD exponentiation simultaneously.

The name HERWIRI acknowledges that the initial versions build upon the HERWIG [12] parton shower generator. The first to be released, HERWIRI1, [11] implemented IR-improved splitting kernels [13] obtained using the QCD analog of YFS exponentiation. The IR-improved kernels have also been implemented [14] in MC@ NLO [15]. The ultimate goal is a complete shower generator based entirely on $\mathrm{QCD} \otimes \mathrm{QED}$ exponentiation with exact $\mathscr{O}\left(\alpha_{s}^{2}, \alpha_{s} \alpha, \alpha^{2}\right)$ residuals. [16]

For electron-positron colliders, precision electroweak corrections have been implemented in the program $K K \mathrm{MC}$ [8], which had a precision tag for LEP2 of $0.2 \%$. KKMC uses YFS [6] exponentiated multiple-photon radiation for both the initial and final state, and includes $\mathscr{O}(\alpha)$ electroweak corrections [17, 18, 19] via the DIZET6.21 [20] package developed for ZFITTER[21]. YFS residuals are calculated perturbatively to the relevant orders in $\alpha^{k} L^{l}\left(L=\ln \left(s / m_{e}^{2}\right)\right)$ and exact collinear bremsstrahlung is implemented for up to three photons.

HERWIRI2[22] implements the electroweak radiative corrections of $K K \mathrm{MC}$ in a hadronic shower generator, presently taken to be HERWIG. $K K \mathrm{MC}$ benefits from a very efficient representation of $n$-photon phase space, with complete control over the soft and collinear singularities for an arbitrary number of photons Real and virtual IR singularities cancel exactly to all orders.

The Drell-Yan cross section with multiple-photon emission can be expressed as an integral over the parton-level process $q_{i}\left(p_{1}\right) \bar{q}_{i}\left(p_{2}\right) \rightarrow f\left(p_{3}\right) \bar{f}\left(p_{4}\right)+n \gamma(k)$, integrated over phase space and summed over photons. The parton momenta $p_{1}, p_{2}$ are generated using parton distribution functions giving a process at CMS energy $q$ and momentum fractions $x_{1}, x_{2}$ such that $q^{2}=x_{1} x_{2}$ s:

$$
\sigma_{\mathrm{DY}}=\int \frac{d x_{1}}{x_{1}} \frac{d x_{2}}{x_{2}} \sum_{i} f_{i}\left(q, x_{1}\right) f_{\bar{i}}\left(q, x_{2}\right) \sigma_{i}\left(q^{2}\right) \boldsymbol{\delta}\left(q^{2}-x_{1} x_{2} s\right),
$$

where the final state phase space includes $p_{3}, p_{4}$ and $k_{i}, i=1, \cdots, n$ and multiple gluon radiation + hadronization is included through a shower.

HERWIRI2 uses HERWIG 6.5[12] as the shower generator, which creates the hard process first at Born level. HERWIRI2 finds the $Z / \gamma^{*}$ and the partons interacting with it in the event 
record. The initial partons define $p_{1}, p_{2}$, which are transformed to the $\mathrm{CM}$ frame and projected on-shell to create a starting point for $K K \mathrm{MC}$, which generates the final fermion momenta $p_{3}, p_{4}$ and photons $k_{i}$ (both ISR and FSR.) The generated particles are transformed back to the lab frame and placed in the event record.

With a change of variables, the Drell-Yan cross section in HERWIG, and thus in HERWIRI2, can be expressed as

$$
\begin{aligned}
\sigma_{\mathrm{DY}} & =\int \frac{d x_{1}}{x_{1}} \frac{d x_{2}}{x_{2}} \sum_{i} f_{i}\left(q, x_{1}\right) f_{\bar{i}}\left(q, x_{2}\right) \sigma_{i}\left(q^{2}\right) \delta\left(q^{2}-x_{1} x_{2} s\right) \\
& =\int_{q_{\min }}^{q_{\max }} d q P(q) \int_{q^{2} / s}^{1} \frac{d x_{1}}{x_{1}} \sum_{i} P_{i} W_{\mathrm{HW}}^{(i)}\left(q^{2}, x_{1}\right)=\left\langle W_{\mathrm{HW}}\right\rangle
\end{aligned}
$$

where $P(q)$ is a normalized, integrable, crude probability distribution for $q, P_{i}$ is the crude probability of generating parton $i$, and $W_{\mathrm{HW}}$ is the HERWIG event weight. This weight depends only on the hard Born cross section and is not altered by the shower.

The crude probability distributions used by HERWIG are

$$
P(q)=\frac{1}{2}\left[P_{\gamma}(q)+P_{Z}(q)\right], \quad P_{\gamma}(q)=\frac{N_{\gamma}}{q^{4}}, \quad P_{Z}(q)=\frac{N_{2} q}{\left(q^{2}-M_{Z}^{2}\right)+\Gamma_{Z}^{2} M_{Z}^{2}}
$$

The HERWIG event weight is

$$
W_{\mathrm{HW}}=\sum_{i} W_{\mathrm{HW}}^{(i)}, \quad W_{\mathrm{HW}}^{(i)}=\frac{1}{P(q)} f_{i}\left(q, x_{1}\right) f_{\bar{i}}\left(q, x_{2}\right) \ln \left(\frac{s}{q^{2}}\right) \sigma_{\mathrm{HW}}^{(i)}\left(q^{2}\right)
$$

and the corresponding probability for selecting parton $i$ is

$$
P_{i}=W_{\mathrm{HW}}^{(i)} / W_{\mathrm{HW}}
$$

Electroweak corrections may be introduced via a form factor

$$
F_{E W}^{(i)}\left(q^{2}\right)=\frac{\sigma_{i}\left(q^{2}\right)}{\sigma_{\text {Born }}^{(i)}\left(q^{2}\right)}
$$

$K K \mathrm{MC}$ will calculate the EW form factor, and multiply it by the HERWIG Born cross section. To avoid double-counting EW effects, any EW parameters in the denominator of eq. (6) must match those in HERWIG. The total cross section may be expressed as the average of a combined weight,

$$
\sigma_{\mathrm{tot}}=\left\langle W_{\mathrm{tot}}\right\rangle, \quad W_{\mathrm{tot}}=F_{E W}^{(i)}\left(q^{2}\right) W_{\mathrm{HW}}=W_{\mathrm{HW}} \frac{\sigma_{\mathrm{KK}}^{(i)}\left(q^{2}\right)}{\sigma_{\mathrm{Born}}^{(i)}\left(q^{2}\right)} .
$$

The $K K \mathrm{MC}$ cross section is calculated using a primary distribution

$$
\frac{d \sigma_{\mathrm{Pri}}^{(i)}(s, v)}{d v}=\sigma_{\mathrm{Born}}^{(i)}(s(1-v)) \frac{1}{2}\left(1+\frac{1}{\sqrt{1-v}}\right) \bar{\gamma}_{i} v^{\bar{\gamma}_{i}-1} v_{\min }^{\gamma_{i}-\bar{\gamma}_{i}}
$$

with

$$
\gamma_{i}=\frac{2 \alpha}{\pi} Q_{i}^{2}\left[\ln \left(\frac{s}{m_{i}^{2}}\right)-1\right], \quad \bar{\gamma}_{i}=\frac{2 \alpha}{\pi} Q_{i}^{2} \ln \left(\frac{s}{m_{i}^{2}}\right)
$$


to generate the factor $v$ giving the fraction of $s$ remaining after ISR photon emission, $s_{X}=s(1-v)$.

The $K K \mathrm{MC}$ cross section is

$$
\sigma\left(q^{2}\right)=\int d \sigma_{\mathrm{Pri}} \frac{d \sigma_{\mathrm{Cru}}}{d \sigma_{\mathrm{Pri}}} \frac{d \sigma_{\mathrm{Mod}}}{d \sigma_{\mathrm{Cru}}}=\sigma_{\mathrm{Pri}}\left\langle W_{\mathrm{Cru}} W_{\mathrm{Mod}}\right\rangle .
$$

$W_{\text {Cru }}$ is calculated during ISR generation and $W_{\text {Mod }}$ is generated after $s_{X}$ is available.

The HERWIG and KKMC weights are combined to calculate the total HERWIRI2 weight,

$$
\sigma_{\mathrm{tot}}=\left\langle W_{\mathrm{HW}} \frac{\sigma_{i}\left(q^{2}\right)}{\sigma_{\mathrm{Born}}^{(i) \star}\left(q^{2}\right)}\right\rangle=\left\langle W_{\mathrm{HW}} \sigma_{\mathrm{Pri}}^{(i)}\left(q^{2}\right) \frac{W_{\mathrm{Cru}}^{(i)} W_{\mathrm{Mod}}^{(i)}}{\sigma_{\mathrm{Born}}^{(i) \star}\left(q^{2}\right)}\right\rangle,
$$

This average will eventually be calculated using a joint probability distribution for $q$ and $v$, $D(q, v)=P(q) d \sigma_{\mathrm{Pri}} / d v$, with $P(q)$ from HERWIG. An adaptive MC (S. Jadach's FOAM [23]) will calculate the normalization of the distribution at the beginning of the run, in a similar manner to how $K K \mathrm{MC}$ presently integrates the one-dimensional primary distribution. However, as a first step, we have constructed a version of HERWIRI 2 using $K K$ MC's one-dimensional primary distribution.

In the present scheme, the built-in primary distribution for electrons at scale $q_{0}=M_{Z}$ is be used for the low-level generation of $v$. The transformation from this distribution to a distribution at HERWIG's generated scale $q$ for quark $i$ is then obtained by a change of variables. The result may be expressed as an average of a product

$$
\sigma_{\mathrm{tot}}=\left\langle W_{\mathrm{HW}} W_{\mathrm{Mod}} W_{\mathrm{Karl}} W_{\mathrm{FF}} W_{\gamma}\right\rangle
$$

with new weights defined by

$$
W_{\mathrm{Karl}}=\frac{\sigma_{\mathrm{Pri}}^{(e)} W_{\mathrm{Crud}}^{(i)}}{\sigma_{\mathrm{Born}}^{(e)}\left(q_{0}^{2}(1-v)\right)}, \quad W_{\mathrm{FF}}=\frac{\sigma_{\mathrm{Born}}^{(i)}\left(q^{2}(1-v)\right)}{\sigma_{\mathrm{Born}}^{(i) \star}\left(q^{2}\right)}, \quad W_{\gamma}=\frac{\gamma_{i}}{\gamma_{e}} \frac{F_{\mathrm{YFS}}^{(i)}}{F_{\mathrm{YFS}}^{(e)}} v^{\gamma_{i}-\gamma_{e}},
$$

with YFS form factors

$$
F_{\mathrm{YFS}}^{(i)}=\frac{e^{-C_{E} \gamma_{i}}}{\Gamma\left(1+\gamma_{i}\right)}, \quad F_{\mathrm{YFS}}^{(e)}=\frac{e^{-C_{E} \gamma_{e}}}{\Gamma\left(1+\gamma_{e}\right)},
$$

and Euler's number $C_{E}=0.5772 \ldots$. The $\gamma$ factors are calculated using $q^{2} / m_{i}^{2}$ for parton $i$ and $q_{0}^{2} / m_{e}^{2}$ for the electron. The weight $W_{\gamma}$ has been modified since the first publication on HEWIRI2[22], and may be modified further, due to the discovery of some uncancelled dependence on $v_{\min }$, a cutoff in eq. (8) which should not affect the final result.

HERWIRI2 is still under development, so any numerical results must be treated as preliminary. A $10^{6}$-event run for $p p$ collisions at $8 \mathrm{TeV}$ with the $Z / \gamma^{*}$ invariant mass bounded by $30 \mathrm{GeV}$ and $300 \mathrm{GeV}$, using HERWIG 6.520 default parameters and CT10 PDFs [24], yields a cross-section of $1218 \pm 13 \mathrm{pb}$, which is a $5.9 \%$ electroweak correction, a reasonable magnitude in light of previous calculations.[2] An average of 0.45 ISR photons and 0.61 FSR photons are generated per event, with average total energies of 0.63 and $1.16 \mathrm{GeV}$, respectively.

Work is in progress to optimize MC generation in the presence of ISR. As noted above, there is still some residual dependence on a cutoff $v_{\min }$ in $K K \mathrm{MC}$, which will require further refinement of the weights. This can be traced to the fixed scale $q_{0}$ in generating the primary distribution for ISR. The best solution of this will probably be to use $K K \mathrm{MC}$ 's beamsstrahlung feature to better 
model the range of parton CMS energies generated by HERWIG. It will be especially interesting to see the effect of initial state radiation, which appears to enter at the $2-3 \%$ level, making it crucial to precision calculations. HERWIRI2 will be an important step toward the goal of a hadronic event generator based on nonabelian $\mathrm{QCD} \otimes \mathrm{QED}$ exponentiation with exact $\mathscr{O}\left(\alpha_{s}^{2}, \alpha_{s} \alpha, \alpha^{2}\right)$ residuals.

\section{Acknowledgments}

S. Yost thanks the organizers of ICHEP 2012 for the invitation to present these results, and D. Marlow and the Princeton Physics Department for their support and hospitality during a critical period of its development. S. Yost and B.F.L. Ward also acknowledge the hospitality of the CERN theory division, which contributed greatly to the progress of this work.

\section{References}

[1] M. Dittmar, F. Pauss, and D. Zurcher, Phys. Rev. D56 (1997) 7284; V.A. Khoze, A.D. Martin, R. Orava and M.G. Ryskin, Eur. Phys. J. C19 (2001) 313; W.T. Giele and S.A. Keller, arXiv:hepph/0104053; M.W. Krasny, F. Fayette, W. Placzek and A. Siódmok, Eur. Phys. J. C51 (2007) 608 .

[2] N.E. Adam, V. Halyo, and S.A. Yost, JHEP 05 (2008) 062 [arXiv:0802.3251]; ibid., JHEP 11 (2010) 074 [arXiv:1006.3766]; N.E. Adam, V. Halyo, S.A. Yost, and W.-H. Zhu, JHEP 09 (2008) 133 [arXiv:0808.0758].

[3] K. Melnikov and F. Petriello, Phys. Rev. Lett. 96 (2006) 231803; Phys. Rev. D74 (2006) 114017; R. Gavin, Y. Li, F. Petriello, and S. Quackenbush, Comput. Phys. Commun. 182 (2011) 2388.

[4] C.M. Carloni Calame, G. Montagna, O. Nicrosini, and M. Treccani, JHEP 05 (2005) 019; C.M. Carloni Calame, G. Montagna, O. Nicrosini, and A. Vicini, JHEP 12 (2006) 016; ibid., JHEP 10 (2007) 109.

[5] E. Barberio, B. van Eijk, and Z. Wạs, Comput. Phys. Commun. 66 (1991) 115; E. Barberio and Z. Wass, Comput. Phys. Commun. 79 (1994) 291; P. Golonka and Z. Wa̧s, Eur. Phys. J. C45 (2006) 97.

[6] D.R. Yennie, S. Frautschi, and H. Suura, Ann. Phys. 13 (1961) 379.

[7] S. Jadach, E. Richter-W as, B.F.L. Ward, and Z. Wạs, Comput. Phys. Commun. 70 (1992) 305;

[8] S. Jadach, B.F.L. Ward, and Z. Wass, Comput. Phys. Commun. 130 (2000) 130. S. Jadach, W. Płaczek, E. Richter-W as, B.F.L. Ward, and Z. Wąs, Comput. Phys. Commun. 102 (1997) 229; B.F.L. Ward, S. Jadach, M. Melles, and S.A. Yost, Phys. Lett. B450 (1999) 262.

[9] S. Jadach, W. Płaczek, and B.F.L. Ward, Phys. Lett. $B 390$ (1997) 298; S. Jadach, B.F.L. Ward, and Z. Wąs, Comput. Phys. Commun. 79 (1994) 503; M. Skrzypek, S. Jadach, W. Płaczek, and Z. Wąs, Comput. Phys. Commun. 94 (1996) 216; S. Jadach, W. Płaczek, M. Skrzypek, B.F.L. Ward, and Z. Wąs, Comput. Phys. Commun. 119 (1999) 272; ibid., Comput. Phys. Commun. 140 (2001) 432.

[10] C. Glosser, S. Jadach, B.F.L. Ward, and S.A. Yost, Mod. Phys. Lett. A19 (2004) 2113; B.F.L. Ward, C. Glosser, S. Jadach, and S.A. Yost, Int. J. Mod. Phys. A20 (2005) 3735; B.F.L. Ward and S.A. Yost, in Proc. ICHEP04, Beijing, vol. 1, ed. H. Chen et al. (World Scientific, Singapore, 2005), 588 [arXiv:hep-ph/0410277]; ibid., Acta Phys. Polon. B38 (2007) 2395; ibid., in Proc. ICHEP06, Moscow, vol. 1, 505 [arXiv:hep-ph/0610230]; ibid., PoS (RAD COR 2007) 038 [arXiv:0802.0724]; ibid.; B.F.L. Ward, S. Joseph, S. Majhi, and S.A. Yost, Proc. 2008 HERA-LHC Workshop, 
DESY-PROC-2009-02, eds. H. Jung, A. De Roeck (DESY, Hamburg, 2009) 180 [arXiv:0808.3133]; ibid., in Proc. ICHEP08, Philadelphia [arXiv:0810.0723].

[11] S. Joseph, S. Majhi, B.F.L. Ward and S.A. Yost, Phys. Lett. $\mathbf{B 6 8 5}$ (2010) 283; ibid., Phys. Rev. D81 (2010) 076008; ibid., Mod. Phys. Lett. A25 (2010) 2207; in Proc. DPF-2009, Detroit eConf C090726 [arXiv:0910.0491].

[12] G. Corcella, I.G. Knowles, G. Marchesini, S. Moretti, K. Odagiri, P. Richardson, M.H. Seymour, and B.R. Webber, HERWIG6.5, arXiv:hep-ph/0011363.

[13] B.F.L. Ward, Adv. High Energy Phys. 2008 (2008) 682312; Ann. Phys. 323 (2008) 2147.

[14] S. Joseph, S. Majhi, B.F.L. Ward and S.A. Yost, PoS (RADCOR2009) 070 [arXiv:1001.2730]; B.F.L. Ward, S. Majhi, and S.A. Yost, PoS (RADCOR2011) 022 [arXiv:1201.0515]; S.K. Majhi, A. Mukhopadhyay, B.F.L. Ward, and S.A. Yost, submitted to Phys. Lett. B (2012) [arXiv:1208.4750].

[15] S. Frixione and B. Webber, JHEP 0206 (2002) 029; S. Frixione, P. Nason, and B. Webber, JHEP 0308 (2003) 007.

[16] S.A. Yost, Chris Glosser, and B.F.L. Ward, in Third International Symposium on Quantum Theory and Symmetries, Cincinnati, 2003 (World Scientific, Singapore, 2004) 775 [arXiv:hep-ph/0401211]; S.A. Yost and B.F.L. Ward, Nucl. Phys. (Proc. Supp.) B157 (2006) 78 [arXiv:hep-ph/0602030].

[17] D. Bardin, P. Christova, and O. Fedorenko, Nucl. Phys. B175 (1980) 435; Nucl. Phys. B197 (1982) 1; A. Akhundov, D. Bardin, and T. Riemann, Nucl. Phys. B276 (1986) 1; D. Bardin, S. Riemann, and T. Riemann, Z. Physik C32 (1986) 121; D. Bardin, S. Bilenky, G. Mitselmakher, T. Riemann, and M. Sachwitz, Z. Physik C44 (1989) 493; D. Bardin, W. Hollik, and T. Riemann, Z. Physik C49 (1991) 485 .

[18] A. Djouadi and C. Verzegnassi, Phys. Lett. B195 (1987) 265; A. Djouadi, Nuovo Cim. 100A (1988) 357.

[19] H. Burkhardt, F. Jegerlehner, G. Pensko, and C. Verzegnassi, Z. Physik C43 (1989) 497; F. Jegerlehner, in Progress in Particle and Nuclear Physics, Vol. 27, ed. A. Fassler (Pergamon Press, Oxford, 1991) 32.

[20] A. Akhundov, D. Bardin, M. Bilenky, P. Christova, S. Riemann, T. Riemann, M. Sachwitz, and H. Vogt, DIZET6.21

[21] A. Akhundov, D. Bardin, and T. Riemann, Phys. Lett. B166 (1986) 111.

[22] S.A. Yost, V. Halyo, M. Hejna, and B.F.L. Ward, PoS (RADCOR2011) 017 [arXiv:1201.5906]

[23] S. Jadach, Comput. Phys. Commun. 130 (2000) 244.

[24] H.-L. Lai, M. Guzzi, J. Huston, J. Li, P.M. Nadolsky, J. Pumplin, and C.-P. Yuan, Phys. Rev. D82 (2010) 074024. 